\title{
Schwankungen im Eiweißgehalt und in der Leitfähigkeit beim Sänglingshlute.
}

\author{
Von \\ cand. med. Otto Hagner (Freiburg i. Br.). \\ (Aus der Universitätskinderklinik Freiburg i. B. [Dir. Prof. Dr. Nalge].)
}

(Eingegangen am 12. April 1913.)

I.

Der Kampf ums Dasein gestaltet sich bekanntlich für den Säugling der ersten Lebensmonate ungleich schwieriger als für den Erwachsenen. Dies erhellt vor allem aus dem Ergebnis der pädiatrischen Forschung der beiden letzten Jahrzehnte, die insbesondere die Mängel und Unfertigkeiten des jugendlichen Organismus zu dieser Zeit klargestellt, jedoch auch die große, rom Säugling zu bewältigende, physiologisch notwendige Stoffwechselarbeit genügend betont hat.

Durchgehend weisen die einschlägigen Untersuchmingen auf die gesteigerten Anforderungen an den Säuglingsorganismus durch die manuigfaltigen Aufgaben, welche unstreitig größer sind als beim älteren Individuum, hin. Der Fähigkeit des Kindes, diesen größeren Ansprüchen zu genügen, sind, wie die Erfahrung lehrt, recht enge Grenzen gezogen, zumal da eine für den Organismus des Erwachsenen unwesentliche Störung für den infantilen Organismus hinreicht, ım die Gesundheit, ja sogar das Leben in Frage zu stellen. Die einzelnen Stoffwechselfaktoren befinden sich eben beim Neugeborenen im labilen Gleichgewicht. Hierbei hängt das leichtere oder weniger leichte Versagen der einzelnen Stoffwechselfunktionen, die eine unzulängliche Vorbereitung für die an sie herantretenden Aufgaben aufweisen, von der Konstitution des Säuglings ab, d. h. von der Anpassungsfähigkeit des Organismus an die Anforderungen des Lebens.

Czerny hob schon bei der Aufstellung seiner Lehre von der exsudativen Diathese diese den einzelnen Individuen in verschiedenem MaBe zukommende Eigenart hervor unter Hinweis auf die durch das konstitutionelle Moment bedingte Beeinflussung des Ernährungsergebnisses ${ }^{1}$ ).

1) Czerny, Exsudative Diathese. Jahrb. f. Kinderheilk. 11, 3. Folge, ‥ 199 ff. 
Er hat mit der Berücksichtigung dieses wichtigen Momentes der Kon" stitution damals gewisses Aufsehen in pädiatrischen Kreisen erregt, ohne daß man jedoch auf diese Anregung hin die Erforschung der wesentlichen Eigenschaften des Säuglingsorganismus zum Gegenstand der Untersuchung gemacht hätte, obgleich die Bedeutung der individuellen Eigenart für die Entwicklung des jugendlichen Organismus wie auch für die Beeinflussung des Ernährungserfolges und des Krankheitsbildes des Kindes nicht verkannt wurde.

Erst eigentlich mein verehrter Lehrer, Prof. Dr. Salge, hat in seiner Antrittsvorlesung an der Universität Freiburg i. Br. über das Thema „Der Kampf ums Leben im Säuglingsalter"1) ron neuem auf das ungemein wichtige Studium der Konstitution des Säuglingsorganismus hingewiesen, das nach seiner Ansicht für das Verständnis der Frnährungs- und Stoffwechselstörungen von ausschlaggebender Bedeutung ist.

Im Mittelpunkt des Interesses steht dabei die Frage nach der Fähig* keit des Säuglings, die es ihm ermöglicht, die physikalischen Eigenschaften seines milieu interne konstant zu erhalten, im besondern nach der ausreichenden Regulation des osmotischen Druckes. Daß auch diese Regulierungsfähigkeit eimen höchst labilen (harakter hat, erhellt aus den gleich zu erwähnenden Untersuchungen, die auf Grund der enomen śchwankungen im Wassergehalt des alimentärkranken Säuglingsorganismus, welche bis zur Entstehung ron alimentärbedingten Ödemen ohne irgenlwelche erkembare Nierenschädigung führen, vorgenommen wurden. Diese enormen Wasserschwankungen, die ebenso beträchtliche Gewichtsschwankungen klärlich zur Folge hatten, waren der klinischen. Erfahrung ja schon längst geläufig. Es bedurfte indes einer einwandfreien Erklärung dieser Wasser- und Gewichtsschwankungen, zumal da sie, wie der klinischen Beobachtung bekannt war, keineswegs durchgehend in ihrem gegenseitigen Verhalten mit der Schwere des klinischen Krankheitsbildes parallel gingen, besonders beim magendarmkranken Säugling. Reiss ${ }^{2}$ ) wies vor wenigen Jahren erneut auf diese großen Schwankungen im Wasserhaushalt in seiner Arbeit iber Untersuchungen ker Blutkonzentration hin und betonte von neuem den Wert des Körpergewichts für die Beurteilung der Ernährung und der einzelnen Nähr-

1) B. Salge, Der Kampf ums Leben im Säuglingsalter. Freiburg i. Br. 1910, ‥ $13 \mathrm{ff}$.

2) E. Reiss, Untersuchungen der Blutkonzentration des Säuglings. Jahrb. f. Kinderheilk. 20, 3. Folge, S. 311. 
schäden, den man zuvor stark in Zweifel gezogen hatte, indem er auf Grund der Möglichkeit des gegenseitigen Verhaltens der Körpergewichtsund Blutkonzentrationskurve beim kranken Säugling und unter Heranziehung des zugehörigen klinischen Verlaufes der Krankheit einen Beitrag zur Deutung der Schwankungen im Wasserhaushalt des jugendlichen Organismus zu geben hoffte. Reiss bediente sich auch bei diesen Bestimmungen der Blutkonzentration wie in seinen früheren Arbeiten der refraktometrischen Methode als der bequemsten und sichersten, um Wasserschwankungen der Körpersäfte zu verfolgen.

Die Verwendung des Refraktoneters setzt für die richtige Deutung der gefundenen Eiweißwerte vor allem die Kenntnis des normalen Eiweißgehaltes des Säuglingsblutserums voraus, und es ist durchaus das Verdienst von Reiss, zuerst die normale Eiweißkonzentration beim Erwachsenen und beim gesunden Säugling in den einzelnen Lebensmonaten bestimmt zu haben, zumal da der normale Eiweißgehalt den Maßstab bei seinen Untersuchungen für die gefundenen Eiweißwerte abgeben mußte, die ihrerseits wieder als Indikator für die Größe der Schwankungen im Wassergehalt des kindlichen Organismus dienen.

Die von ihm untersuchten gesunden Kinder zeigen bis zum 6 . Lebensmonat einen durchschnittlichen Eiweißwert ihres Blutserums von etwa $6 \%$; somit wäre also der Eiweibgehalt des Säuglings um etwa $2 \%$ niedriger als der des Erwachsenen mit 7,5--9\%. Es sind demnach die Körpersäfte des Säuglings wesentlich wasserreicher als die des älteren Kindes und des Erwachsenen. Diesen normalen Eiweißwert von ca. $6^{\circ}$ habe ich meinen refraktometrischen Untersuchungen zugrunde gelegt.

Die Zeit vom 6 . bis 10. Monat im Säuglingsalter ist vor allem gekennzeichnet durch die größere Selbständigkeit des jugendlichen Organismus gegenüber äußeren Lebensbedingungen; es ist zugleich die Zeit des Uberganges zur Nahrung des älteren Kindes, d. h. die arteigene Nahrung tritt mehr und mehr in den Hintergrund und wird ersetzt durch die gemisehte Kost. In diese Zeit des Nahrungswechsels fällt auch nach den Reissschen Angaben das Ubergangsstadium der Blutkonzentration des Säuglings zu der des Erwachsenen. Der Eiweißwert beträgt von jetzt ab $7,5-9 \%$.

Auch hieraus erhellt deutlich, daß der Neugeborene über einen höchst unfertigen, erst im Werden begriffenen Organismus in der ersten Lebenszeit verfügt.

Außer dem Eiweißgehalt des Blutserums kommt noch ein zweiter Faktor, der relativ geringe Salzgehalt, hinzu, ron dem der osmotische 
Druck in der Hauptsache abhängig ist. Jedoch auch der Salzgehalt des Organismus ist nicht stabiler Natur, sondern der Beeinflussung durch alimentäre Störungen unterworfen, wie dies auch die Arbeiten von Steinitz $z^{1}$, Weigert ${ }^{2}$ ) und namentlich ron L. F. Meyer ${ }^{3}$ ) betonen.

Berücksichtigt man die oben erwähnten enormen Wasserschwankungen beim alimentärkranken Säugling. so liegt der Gedanke nahe, daB sie in ursächlichem Zusammenhang stehen könnten mit der mangelhaften Fähigkeit des Säuglingsorganismus, seinen osmotischen Druck zu regulieren, ihn konstant zu halten. Gewiß haben die oben erwähnten Arbeiten die Beeinflussung des Salzgehaltes durch pathologisch-alimentäre Vorgänge nicht außer acht gelassen, ohne jedoch die enormen Schwankungen. im Wasserhaushalt auf die unzulängliche Regulationsfähigkeit des osmotischen Truckes rles jugendlichen Organismus zu beziehen. Seine Konstanterhaltung galt eben durchaus als feststehende Regel, ohne daß man bedacht hätte, flaß dem Säugling damit ein größeres Regulationsvermögen zugestanden wird als dem Erwachsenen ${ }^{4}$ ).

Auch ReiB legt dem geringen Salzgehalt des Blutserums keine weitere Bedeutung für die Wasserschwankungen bei, da auch er damals dieser Meinung war: .,Der osmotische Druck bleibt also im allgemeinen unverändert oder ändert sich nur sehr wenig. ${ }^{* 5}$ )

Erst der geeigneten. einwandfreien und neu ausgearbeiteten phyikalischen Methode der Gefrierpunktsbestimmung ron Salge ${ }^{6}$ ), die ror allem nur kleine Serummengen zur Thtersuchung benötigt, blieb es rorbehalten, die Schwankungen des osmotischen Druckes nachzuweisen und damit eine Erklärung für die Schwankungen im Wasserhaushalt zu geben. Sein früherer Schluß, ,daß vielmehr eine Wechselbeziehung zwischen Stoffwechselstörung und der Mangelhaftigkeit dieses Regulationsvorganges existiere ${ }^{67}$ ), wurde durch seine Untersuchungen bestätigt.

1) Siteinitz. Über den Einfluß von Ernährungsstörungen auf die chemisehe Zusammensetzung des Säuglingskörpers. Jahrb. f. Kinderheilk. 59, S. 450ff.

$\left.{ }^{2}\right)$ Weigert, C̈ber den Einfluß der Ernährung auf die chemische Zusammensetzung des Organismus. Jahrb. f. Kinderheilk. 11, 3. Folge, S. $178 \mathrm{ff}$.

$\left.{ }^{3}\right)$ L. F. Meyer, Zur Kenntnis des Stoffwechsels bei den alimentären Intoxikationen. Jahrb. f. Kinderheilk. 65, S. $585 \mathrm{ff}$.

4) Vgl. B. Salge. Die physikalischen Erscheinungen des Blutes beim gesunden und kranken säugling. Zeitsehr. f. Kinderheilk. 1910, S. $128 \mathrm{ff}$, Mitteilung I.

5) Vgl. E. Reiss, a. a. O., S. 362.

5) Vgl. B. Sialge, a. a. O., \$. $131 \mathrm{ff}$.

7) Vgl. B. Sialge, a. a. O., s. 23. 
In seiner Arbeit nahm Reis hauptsächlich zu der Frage Stellung, ob die klinisch beobachteten Gewichtsschwankungen beim kranken Kinde ihre Erklärung, wie man annahm, in der Veränderung in Wassergehalt der Körpersäfte finden oder im Verein mit bestimmten klinischen Erscheinungen durch Hydrämie bedingt sind. Zum Vergleich der refraktometrisch gewonnenen Eiweißwerte, gegeben durch die relativ in der Mehrzahl vorhandenen Eiweißkörper mit Einschluß aller im Blutserum gelösten Substanzen, zog er das Verhalten im Körpergewicht des Säuglings heran und kam auf Grund seiner mit großer Sorgfalt ausgeführten Reihenuntersuchungen beim Säugling zu dem folgenden $\mathrm{Er}$ gebnis:

Der Eiweißgehalt des Blutserums gesunder Ammenkinder bleibt innerhalb normaler Grenzen beinahe konstant, während das Körpergewicht langsam, aber stetig ansteigt, also ein normales Verhalten aufweist.

Bei stärkeren Schwankungen in der Blutkonzentration zeigt, wie ich oben (S. 51) schon andeutete, das Körpergewicht ein wechselvolles Bild in seinem Verhalten, was sich nach der vergleichenden Gewichtsbestimmung von Reiss in dem verschiedenen Verlauf der Körpergewichts- und der Blutkonzentrationskurve kundgibt.

Eine besondere Bedeutung der ganz beträchtlichen Schwankungen im Wasserhaushalt des magendarmkranken Säuglings kommt der das Leben des Kindes bedrohenden, häufig zutage tretenden Bluteindickung besonders bei Cholera infantum zu, die durch Wasserverlust infolge stark einsetzender Diarrhöen bedingt ist. Es versteht sich von selbst, daß damit ein relatives Ansteigen des refraktometrischen Eiweißwertes verbunden ist. Die Eiweißkörper erfahren hierbei keine nachweisbare V'ermehrung in ihrem Bestand.

Die Zusammensetzung der Blutflüssigkeit weist dagegen bei chronischen Verdauungsstörungen und der echten Atrophie meist keine beträchtlichen Veränderungen weder im Wasser- noch im Eiweißgehalt auf. Fehlen akute Darmstörungen, so pflegt in den meisten Fällen sich ein der Norm entsprechender und konstanter Eiweibgehalt des Blutserums einzustellen. Dabei hängt die größere oder geringere Störung naturgemäß von der Beschaffenheit der guten oder schlechten Konstitution des einzelnen Individuums ab.

Im Gegensatz zu der durch Wasserverlust bedingten Bluteindickung steht die durch echte Hydrämie hervorgerufene Blutverdüunung, welche auf Wasserretention durch Niereninsuffizienz oder Kochsalzretention be- 
ruht und eine absolute Wasserzunahme des Blutserums darstellt gegenüber der durch Eiweißabnahme vorgetäuschten Hydrämie. Reiss berichtet in seiner Arbeit nur von zwei Fällen stärkerer Grade echter Hydrämie, die vermutlich mit einer erheblichen Nierenschädigung und mit beträchtlicher Herabsetzung des Eiweißgehaltes zusammengingen. Leichtere Grade der eben genannten echten Hỵdrämie beruhen nach seiner Ansicht wahrscheinlich auf überreichlicher Flüssigkeitszufuhr bei akuter Gastroenteritis. Solche erhebliche Blutverdünnungen haben bekamntlich eine rapide Cewichtszmahme zufolge, die sich jedoch nur zu bald als eine trügerische erweist.

Das Ergebnis all der bisherigen Untersuchungen der Regulierungsfähigkeit der physikalischen Eigenschaften des milieu interne läßt sich somit dahin zusammenfassen, daß der Säuglingsorganismus zur Zeit der extrauterinen Abhängigkeit bei der geringfügigsten Ernährungsstörung oder sonstigen Schädigungen dieser Aufgabe keineswegs gewachsen ist. zumal da die erwähnte Regulationsfähigkeit zu dieser Zeit er st in der Entwicklung begriffen ist.

Die relative Unfähigkeit des kindlichen Organismus, seine Blutkonzentration konstant zu erhalten, ihre Abhängigkeit von der Art der Nahrung. ob arteigene oder künstliche Nährflüssigkeit dem Kinde geboten wircl, all diese Cmstände legen den Gedanken nahe, zu untersucheu. ob diese Schwankung im Eiweißgehalt, im osmotischen Druck und in rer Leitfähigkeit der Körpersäfte auch im Verlaufe des Tages - abhängig von den einzehnen Tageszeiten - bei den einzelnen Individuen rorkommen.

Es ist wohl entschieden von Wichtigkeit zu erfahren, inwieweit dabei die Resorption der Nahrung auf die Konstanterhaltung der Blutkonzentration von Einfluß ist; $m$. a. W. es erhebt sich die Frage, ob der Organismus des Neugeborenen über rle Fähigkeit verfügt, trotz der einzelnen Mahlzeiten während des Tages seine physikalische Zusammensetzung des Blutserums genïgend zu regulieren, d. h. sie möglichst konstant zu erhalten, oder ob beträchtliche Schwankungen auch bei gesunden Sänglingen vorkommen und wie diese Ausschläge zu deuten sind.

Die Anregung zu diesen Untersuchungen über das Verhalten der Regulierungsfähigkeit seines milieu interne des Säuglingsorganismus in den einzelnen Tageszeiten verdanke ich meinem verehrten Lehrer, Herrn Prof. Dr. Salge; für seine wohlwollenden Ratschläge und sein freundliches Entgegenkommen sage ich ihm auch an dieser Stelle herzlichsten Dank. 
II.

Dieser Arbeit liegen 18 Fälle aus der Freiburger Kinderklinik zugrunde, und zwar untersuchte ich das Blutserum der Kinder ohne vorherige Kenntnis der zugehörigen Krankengeschichten usw. auf das Verhalten der lichtbrechenden Substanzen und ihrer Leitfähigkeit zweimal am Tage während des Hungerzustandes und nach der Resorption der Nahrung; darunter einige Säuglinge mehrmals, solange sie in der Klinik anwesend waren. Nach Abschluß der Untersuchungen wurden die erlangten Resultate mit den zugehörigen Krankengeschichten verglichen und die naheliegende Einteilung nach gesunden Säuglingen und kranken Kindern in der Zusammenstellung nach einzelnen Krankheitsbildern getroffen. Die Versuchsdaten sind in der beiliegenden Tabelle zusammengestellt.

1. Zunächst sei das Untersuchungsergebnis mit den zugehörigen Krankengeschichten von drei gesunden Ammenkindem wiedergegeben.

Fall 1. Hedwig H., geb. 2, I. 1913; angeblich 20 Tage übertragen.

Aufgenommen: 15. I. Untersucht: 6 . II.

Anamnese: Gesundes Ammenkind. Mutter hat verengtes Becken. Erstes Kind, kein Abort.

Status praes.: Kleines $(49 \mathrm{~cm})$, rosiges, agiles Kind. Finige Sichrunden auf Stim und Nase, daneben eine Spur von Wangenekzem. Geringer Intertrigo, rotgescheuerte Fersen. Nabel in Ordnung, keine Drïsensehwellumgen. Innere Organe: o. B. Temperatur normal.

Alter: 1 Monat. Gewicht 6. II.: $3260 \mathrm{~g}$. Nahrung: Muttermilch $520 \mathrm{~g}$. Befund: Pirquet negativ. Temperatur $36,4^{\circ}-36,8^{\circ}$. Stühle: 2.

Die Untersuchung des Blutserums ergibt:

6. 1 I.

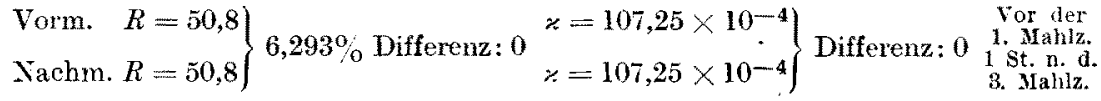

Fall 2. Marie Z., geb. 3. I. 1913. Aufgenommen: 4. II.; untersucht: 6. II.

Anamnese: Gesundes Ammenkind. Erstes Kind, spontan geboren. Brust bekommen.

Status praes.: Kleines, zartes, blasses Kind. Starker Intertrigo. Sonst ist. die Haut sauber, Lunge und Herz: $o$. B. Mundhöhle sauber. Leber und Milz nicht sicher fühlbar. Temperatur normal: $36,6^{\circ}-37,2^{\circ}$.

Alter: 1 Monat. Gewicht 6. II.: 3020 g. 5. II.: 3000 g, Nahrung: Frauenmilch: $420 \mathrm{~g}$. Befund: Pirquet negativ.

Die am 6. II. vorgenommene Untersuchung ergibt:

$\left.\begin{array}{l}\text { Vorm. } R=53,6=6,898 \% \\ \text { Nachm. } R=55,3=7,265 \%\end{array}\right\} \mathrm{D}=-0,367 \% \begin{aligned} & *=108,63 \times 10^{-4} \\ & x=108,18 \times 10^{-4}\end{aligned} \mid \begin{aligned} & \text { Vor d. 1. Mahlzeit } \\ & \mathrm{D}=+0,414^{\circ} \\ & \text { ISt. n. d. 3. Mahlzeit. }\end{aligned}$ 
Fall 3. Anna K., geb, 14. IX. 1912, Frauenklinik. Aufgenommen: 21. IX.; luntersucht: 14., 18. u. 23. I. 1913.

Anamnese: Großeltern bds. gesund. Eltern gesund. Erstes Kind, spontan yeboren.

Status praes.: Kräftiges, wohlgebildetes Kind. Hautfarbe rosig. Kein Ikterus. Hautturgor gut.

(iuter Panniculus. Bauch im Niveau des Thorax. Bauchdecken straff. Mundhöhle o. B. Keine Hals-, kleinste Axillardrüsen. Lunge und Herz: o. B. Leber am Rippenbogen, Milz nicht fühlbar.

Verlauf: 24. IX. 1912. 6 mal Brust. Schläft meist, ist ruhig und zufrieden.

26. IX. Trinkt sehr langsan, daher sehr lange: $8 \mathrm{mal}$ 15-20 Minuten.

27. IX. Geringe eiterige Sekretion aus dem rechten Auge. Therapie: Borwasser. Nahrung: 3 mal Brust und 3 mal abgedrückte Mutternilch. Die Mutter befindet sich in der Hautklinik.

28. IX. Conjunctivitis. Auf dem reehten Auge ist das Nehen besser. Stuhl gelb, homogen.

4. X. Rechtes Auge o. B.

8. X. Hat jetzt 3 mal Brust und 1 mal abgedrückte Frauenmileh; 1 mal Buttermilch. Zunahme gering. . Befinden und Stuhl gut.

18. X. Unruhige Temperatur. Gaumenbögen und Tonsillen gerötet. Rechts eine Occipitaldrüse, bds. kleinste Halsdrüsen jetzt fühlbar. Sonst: o. B.

3. XI. Trinkt schlecht an der Brust, daher 4 mal Brust in 2 mal Flasche 1/1 M., da auf Buttermilch häufig Erbreehen eintritt.

18. XI. Zunahme noch schwankend, trotzt größeren Nahrungsmengen.

28. XI. Trinkt besser, nimmt besser zu, sieht rosig aus. Nettes Fettpolster.

27. XII. Etwas Temperatur, Schnupfen und Husten.

Alter: 4 Monate. Gewicht 14. IX.: 2700 g. Nahrung: 21. IX. 1912 bis 23. I. 1913: Frauenmileh - ganz wenig Buttermileh - I. II. mit Schleim und $5 \%$ Nährzucker - I. I. mit Schleim und $5 \%$ Nährzucker - natürliche Ernährung. Befund: 14. I. 1913: Schlechter getrunken, Schnupfen. Therapie: Protargol. Augenspülung nit Borwasser, Zinktropfen.

Die am 14, 18. u. 23. I. vorgenommenen Untersuchungen ergeben:

14. I.:

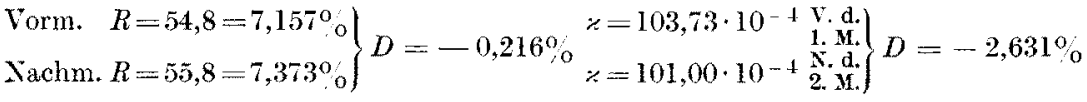

18. I.:

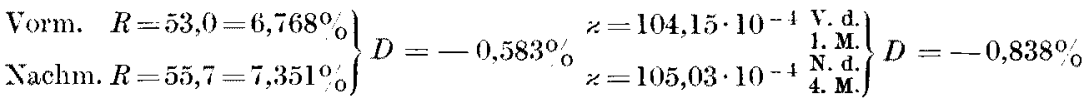
23. I.:

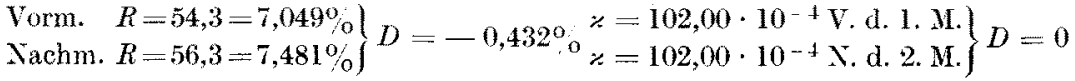

Eine kritische Betrachtung dieser.drei Fälle zeigt zunächst mit Ausuahme des nomalen refraktometrischen EiweiBgehaltes von Fall 1 einen relativ hohen Wert der lichtbrechenden Substanzen, die für eine 
Tabelle.

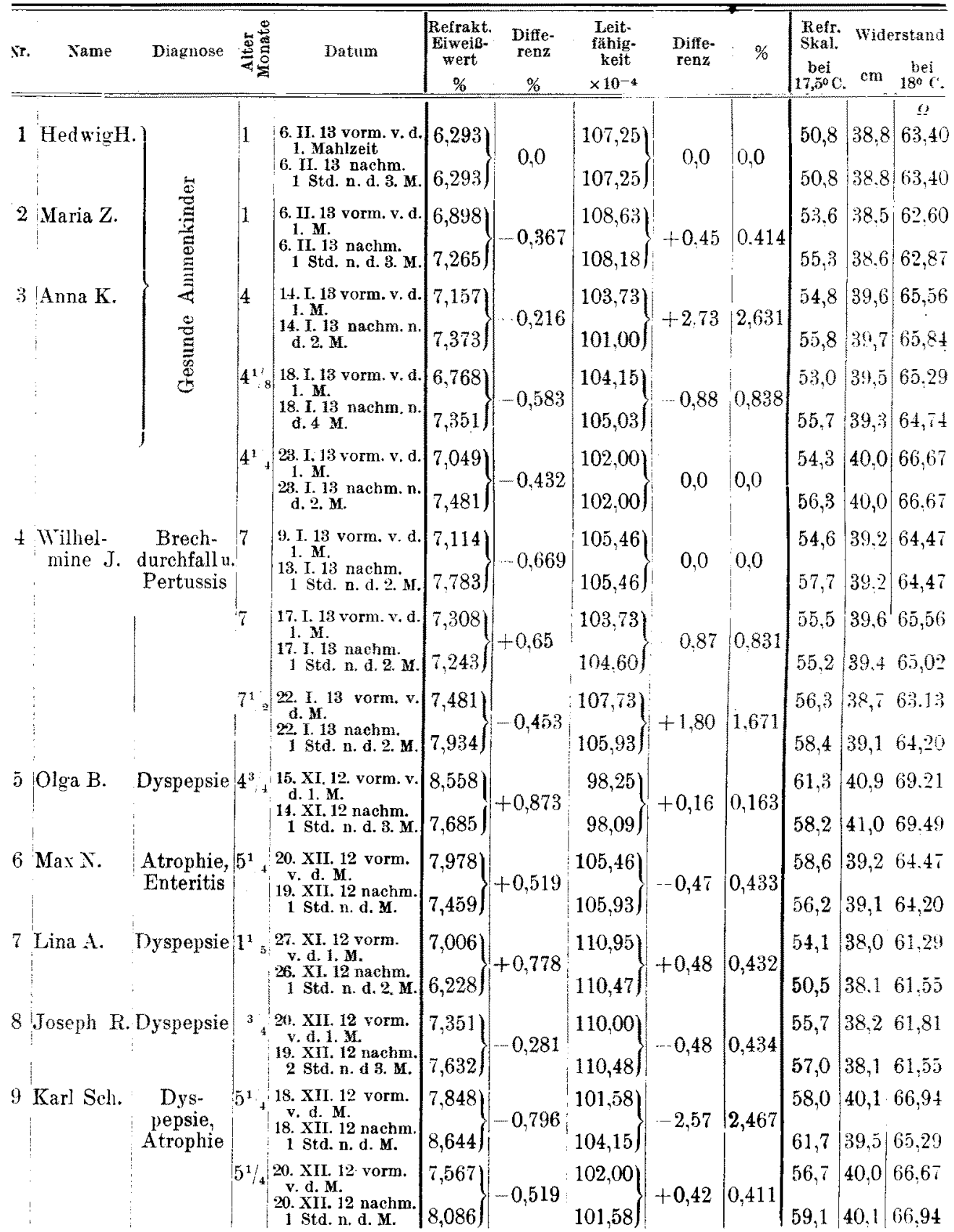


Fortsetzung.

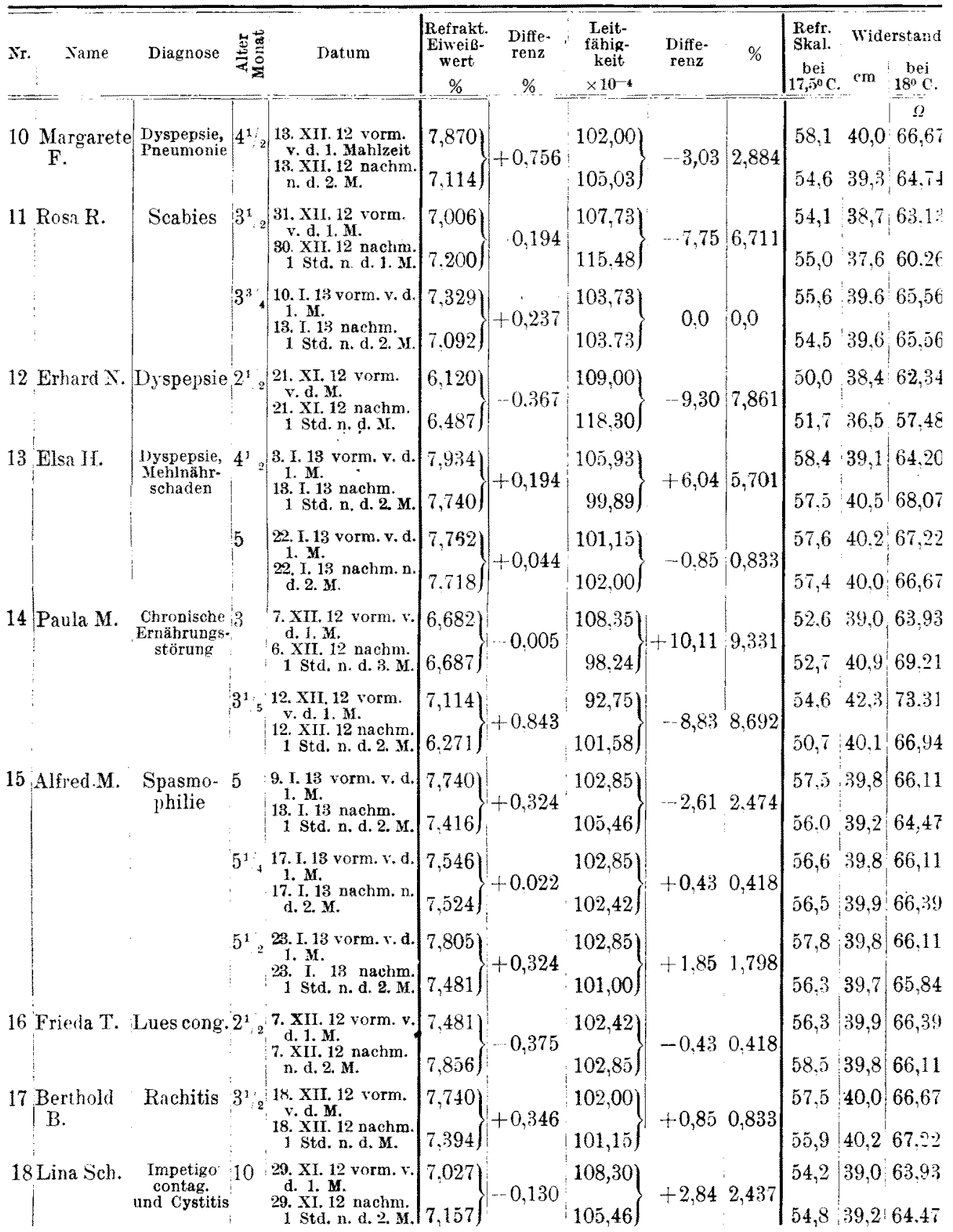


geringfügige Eindickung des Blutes spricht; indessen ist dies kein pathologisches Verhalten der Blutkonzentration, da solche Werte bei sehr jungen Kindern durch mangelhafte Wasseraufnahme vorzukommen pflegen. Ein schneller Ausgleich kommt dann durch genügende Wasseraufnahme zustande. Von Interesse ist wohl das refraktäre Verhalten der Eiweißßwerte im Fall 2 und 3, jedoch sind die Ausschläge viel zu gering, als da $B$ man ihnen irgendwelche Bedeutung beimessen könnte. Somit ist der Eiweißgehalt nach der Resorption etwas höher gestellt als zuvor.

Die Leitfähigkeit entspricht in Fall 1 und 2 nicht den normalen (trenzwerten (100-104.10-4. Salge); die Zahlen für sie sind zu hoch; ries erhellt aus der Tatsache, daß der gesunde Neugeborene mit Wasser nicht entsprechend Salz ausscheidet. Bei genügender Wasseraufnahme kommen normale Zahlen wieder zustande.

Größere Tagesschwankungen in der Leitfähigkeit konmen nur in Fall 3 vor, jedoch innerhalb der gestatteten Variationsbreite. Der Säugling hat an jenem Tage laut Krankenbericht schleehter getrunken und war zudem vom Schnupfen befallen, worin diese Schwankung begriindet sein diurfte. Fall 3 konnte dreimal auf seine Blutkonzentration untersucht werden, ohne daß größere Schwankungen vorgekommen wären.

2. Die folgenden Fälle sind, wie schon erwähnt, in Gruppen nach clen zugrunde liegenden Krankheitsbildern zusammengestellt. Fis folgen zunächst 11 Kinder mit Verdauungs- und Ernährungsstörungen. Sie stellen das größte Kontingent für die pathologischen Fälle der von mir untersuchten Kinder.

Fall 4. Wilhelmine J., geb. 16. VI. 1912. Aufgenommen: 28. XI.; untersucht: 9., 13., 17. u. 22. I. 1913.

Alter: T Monate. Diagnose: Kong. KlumpfüBe, Pertussis, Brechdurchfall, Fruährungsstörung, Cystitis.

Anamnese: ?

itatus: 28. IX. 1912. (Newicht: 3630.g.

In der chirurg. Klinik wegen kongenitaler Klumpfüße in Behandlung. Kein Durchfall und kein Erbrechen. Mageres, elendes Kind. Dicker Bauch. Ganz lebhaft.

Verla uf: 28. XII. $4^{\prime}$ Sichleim $+4^{\prime}$ Milch, 3 stündl., nachts nichts. Gewicht: $3540 \mathrm{~g}$. Keuchhusten soit 4 Wochen. Am 20. XII.: Brechdurchfall. Seit 8 Tagen alend.

A ufnahme: Elendes, zurückgebliebenes Kind. BlaB, schlechte Stimmung. ipastiseher Husten. Klumpfüße. Mund sauber, Rachen frei. Herz o. B. Longe: diffuses Giemen. Bauchdecken papierdünn. Milz und Leber nicht palpabel. Urin: Alb. negatir, massenhaft Leukocyten. Stuhl schleimig.

Verlauf: 6. I. 1913. Nimmt zu. 9. I.: Hustenanfälle mit statkem Rotwerden, sehr suspekt auf Pertussis. 
Nahrung: Abgedrückte Frauenmilch: $400 \mathrm{~g}$., Buttermilch: $350 \mathrm{~g}$,

Befund: 9. 1. 1913. Stuhl: 1; Gewicht: $3600 \mathrm{~g}$; Temperatur: $36,2^{\circ}$. - 13. I. Temperatur: $36,2-37,2^{\circ}$; Gewicht: $3610 \mathrm{~g}$; Stuhl: 1; Hustenstöße: 15.-17. I. Gewicht: $3650 \mathrm{~g}$; Stuhl: 1; HustenstöBe: 16 . -22 . I. Gewicht: $3780 \mathrm{~g}$.; Hustenstöße: 11; Stuhl: 1. Nahrung: Abgedrückte Frauenmilch: 300 g., Buttermilch: $500 \mathrm{~g}$.

Pirquet: - . Therapie: 3 mal Crotrop. : 0,5; 2 mal 1 Pulver Salol; Darmspülung.

Die Untersuchung des Blutserums ergibt folgende Daten für:

9./13. I.:

$\left.\left.\begin{array}{l}\text { Vorm. } R=54,6=7,114 \% \\ \text { Nachm. } R=57,7=7,783 \%\end{array}\right\} \mathrm{D}=-0,669 \% \begin{array}{r}\%=105,46 \cdot 10^{-1} \mathrm{~V} . \text { d. 1. M. } \\ \%=105,46 \cdot 10^{-1} \text { I t. n. d.2. M. }\end{array}\right\} \mathrm{D}=0$ 17. I.:

$\left.\left.\begin{array}{l}\text { Vorm. } R=5 \overline{5}, 5=7,308 \% \\ \text { Nachm. } R=55,2=7,243 \%\end{array}\right\} \mathrm{D}=-0,65 \% \% \begin{array}{l}\%=103,73 \cdot 10^{-4} \\ \%=104,60 \cdot 10^{-4}\end{array}\right\} \mathrm{D}=-0,831 \% \% \begin{aligned} & \text { 1. d. } \\ & \text { i. st. n. } \\ & \text { d. 2. y. }\end{aligned}$ 22. 1. :

Vorm. $\left.R=56,3=7,481^{\circ} \% \mathrm{D}=-0,453 \% \%=107,73 \cdot 10^{-1}\right\} \quad$ For $\mathrm{d}$. Nachm. $\left.R=58,4=7,934_{\%}^{\circ}\right\} \mathrm{D}=-0,453 \% \%$

Am 22. I. 1913 ist in diesem Falle der Wert der lichtbrechenden Substanz dem Alter entsprechend gut. Am 9. I. und 22. I. ist der Eiweißgehalt nach der Resorption höher gestellt als im Hungerzustand. Die Leitfähigkeit ist durchstehend gut. Es ist kein Verlust an dissoziabler Substanz eingetreten. Am 22. I. beträgt die Leitfähigkeit vor der Mahlzeit $107,73 \cdot 10^{-4}$, um aber nachmittags sich mehr der normalen Grenze $104,00 \cdot 10^{-4}$ zu nähern. Die Differenz der Eiweiß-und Leitfähigkeitswerte sind so geringfügig, daß von einer Schwankung nicht gesprochen werden kann.

Fall 5. Olga B., geb. 24. VI. 1912. Eintritt: 11. XI. 1912; untersucht: 15. XI. 1912.

Alter: $4^{3} / 4$ Monate. Diagnose: Cystitis, Dyspepsie, Bronchitis, Pemphigus. Gewicht: $3700 \mathrm{~g}$ am 19. VIII.

Anamnese: 19. VIII. Seit 4 Tagen Durchfall. Hat fichweizer Büchsemmilch crhalten. Während des Durchfalls Eiweiß. In jeder Windel Stuhl.

Status praes.: Kind etwas abgemagert. Turgor noch gut. Befinden ebenfalls gut.

Anamnese: 24. X. Seit 2 Tagen Durchfall und Erbrechen. Großer Bauch. Roter Hals.

Therapie: Sichleim.

Aufnahme: 11. XI. Soll fortwährend schreien und Durchfall haben. Bekam Hileh mit Schleim.

Status praes.: Gewicht : $\mathbf{4 1 9 0} \mathrm{g}$. Stark zurückgebliebenes Kind in schlechtem Ernährungszustand. Fontanelle fast geschlossen. Einzelne Nachtschweiße. Wenig Soor. Rachen frei. Lungen und Herz: o. B. Bauch leicht aufgetrieben. Bauchdeckenspannung leidlich. Milz und Leber nicht palpabel. Stuhl dünn.

Verlauf: 13. XI. Nimmt ab, bricht. Gewicht: 4000 g. Hag die Fiweißmilch nicht, daher ausgesetzt. Trinkt besser. - 17. XI.: Nimmt leidlich zu, trinkt ganz 
gut. Gewicht: $4200 \mathrm{~g}$. Im Urin: Leukocyten. - 22. XI. Normales Befinden. Stuhl geregelt. Gewicht: $4330 \mathrm{~g}$. Mit Urotropin entlassen.

Nahrung: Eiweißmiloh und Tee; I. I. mit Schleim und $5 \%$ Nährzucker. 15. XI.: I. I.: $710 \mathrm{~g}$; 14. XI.: I. I.: $720 \mathrm{~g}$. - Gewicht: I5. XI.: $4100 \mathrm{~g}$; 14. XI.: 4040 g. - Temp.: $36,5^{\circ}-38^{\circ}$ am 15. XI. 14. XI.: $37^{\circ}-36,6^{\circ}$. - Stühle: 15. XI.: 4 ; 14. XI.: 1 .

Die Untersuchung des Blutserums am 15./14. XI. ergibt: $\left.\left.\begin{array}{c}\text { Vorm. } R=61,3=8,558 \% \\ \text { Xachm. } R=58,2=7,685 \%\end{array}\right\} \mathrm{D}:+0,873 \% \% \begin{array}{c}\varkappa=98,25 \cdot 10^{-4} \\ \%=98,09 \cdot 10^{-4}\end{array}\right\} \begin{gathered}\text { Vor d. 1. Y. } \\ \begin{array}{c}*+163 \% \\ \text { I.t. n. d. 3. Y. }\end{array}\end{gathered}$

Das Kind zeigt, wie aus der Krankengeschichte ersichtlich, zur Zeit der Untersuchung vor allem dyspeptische Erscheinungen; am 15. XI. fand durch 4 Stühle ein ziemlich großer Wasserverlust statt, daher die mäßige Eindickung des Blutes. Mit der Wasserabnahme geht ein Verlust an dissoziabler Substanz einher, wie der niedrige Wert für die Leitfähigkeit zeigt. Schwankungen während des Tages in der physikalischen Zusammensetzung des Blutes kommen nicht in Betracht.

Fall 6. Max X., geb. 15. VII. 1912. Eintritt: 29. X. 1912.

Alter: $5^{1} / 4$ Monate. Gewicht: 3540 g. am 29. X. Diagnose: Atrophie, Enteritis.

Anamnese: Bruder hier mit Tuberkulose. Pat. ist ausgetragen, hat keine Brust bekommen. Milch-Reisschleim 3stdl., nachts 1 mal laut Ziehkarte. Gewicht am 1. X.: 3520 g., am 8. X.: 3650 g. Stuhl oft grün. Hustet, hatte Fieber.

Status praes.: Blaß, mager, klein, doch immer noch ganz leidlichen Turgor, Bauchdeckenspannung ganz gut. Muskulatur leidlich. Kleinste Drüsen. Lungen and Herz: o. B. Leber eben am Rippenbogen. Milz nicht fühlbar. Intertrigo. Rachen etwas rot. Auf beiden Tonsillen kleinste Eiweißstippchen.

Ord.: Tee, $1 / 3$ Mileh ohne Zucker.

Verlauf: 4. X. Aussehen Verschlechterung bei Eiweißmileh. Granes Aussehen. Stühle schlecht und gehäuft, bricht. Bauchdeckenspannung bereits schlechter. Turgor noch leidlich. - 5. X. Eiweißmilch stark erbrochen, sehr schlechtes Aussehen. Stühle sehr schlecht, nimmt ab; umgesetzt deshalb auf Frauenmilch. - 9. X. Stühle besser. Aussehen schlecht. Der Gewichtsabfall scheint stehen zu bleiben. - 15. XI. Aussehen unverändert; schlechte Stühle. 14. XI. Stuhl einmal blutig-schleimig. Versuch mit $400 \mathrm{~g}$. Frauenmileh und $200 \mathrm{~g}$. Buttermilch. Darmspülungen mit Tannin. - 16. XI. Einmal stark blutig-schleimigen Stuhl. Bakteriologischer Befund negativ. - 23. XI. Kein Blut im Stuhl. Etwas besser. Gewicht schwankend. - 25. XI.' Kein Blut im Stuhl. Stuhl recht schleimig. - 9. XII. Stühle wechselnd gut und schlecht; kein Blut mehr. Elendes Aussehen. Pirquet 4 mal negativ. - 13. XII. Aussehen immer schlechter werdend, grau, spitz. Stühle wechselnd. Kein Befund. Hier und da Temperaturerhöhung. Hals etwas gerötet. -18 . XII. Tuberkulininjektion $0,0001 \mathrm{~g}$. subcutan $=$ negatir.

Nahrung 29. X. bis 20. XII. 1912: Tee, I. II. mit Schleim ohne Nährzucker, EiweiBmilch, $3 \%$ Xährzucker, $3 \%$ Haferschleim, Frauenmilch, I. I. Am 20. XII.: Abgedrückte Frauenmilch: 200 g., I. I : 300 g., I. II.: 300 g. - 19. XII: Abgedrückte Frauenmilch: 400 g., I. I.: 100 g., I. II. : $300 \mathrm{~g}$. 
Temperatur: 20. XII.: $36,6^{\circ}, 19$. XII.: $37^{\circ}-36,6^{\circ}$. Stühle: 20 . XII.: 3, 15 . XII : 3. Gewicht: 20. XII.: 3440 g.; 19. XII.: 3440 g.

Therapie: Darmspülung mit Tannin. Halsprießnitz. Tuberkulin 0,0001 g. Wärmeflaschen.

Die Untersuchung des Blutserums am 20. XII. ergibt:

$\left.\begin{array}{l}\text { Vorm. } R=58,6=7,978 \% \\ \text { Nachm. } R=56,2=7,459 \%\end{array}\right\}$ D: $\left.+0,519 \% \begin{array}{r}\varkappa=105,46 \cdot 10^{-4} \text { Vor d. M. } \\ \varkappa=105,93 \cdot 10^{-4} 1 \text { St.n.d.M. }\end{array}\right\}$ D:-0,433\%

Hier tritt uns ein Fall von Atrophie entgegen. Das Kind hatte zur Zeit der Untersuchung Stühle von ganz wechselndem Aussehen. Durch den dadurch bedingten Wasserverlust kann der hochgestellte Eiweißwert seine Erklärung finden. Zur Zeit der Resorption ist eine Besserung der Blutkonzentration zu verzeichnen. Nach der guten, normalen Leitfähigkeit $\mathrm{zu}$ urteilen, ist mit dem Wasserverlust keine dissoziable Substanz verlorengegangen. Die Schwankungen in den einzelnen Tageszeiten sind irrelevant.

Fall 7. Lina A., geb. 21. X. 1912. Eintritt: 19. XI.; untersucht: 27. XI. Alter: 5 Wochen. Gewicht: $2870 \mathrm{~g}$. Diagnose: Dyspepsie.

Anamnese: Pflegekind, wegen starker Gewichtsabnahme in die Kinderklinik gebracht und aufgenommen worden.

Status: Stuhl in Ordnung; Pat. bekam Milch-Schleim. Der Mund wurde ausgewischt.

Kleines, recht elendes Kind. Schlechter Krnährungszustand. Graue, blasse Hautfarbe. Soor an der Wangenschleimhaut und am harten Gaumen. Mundhöhle dunkelrot. Lungen und Herz: o. B. Bauch leicht gespannt. Milz nicht palpabel. Starker Intertrigo inguinalis, wund am After. Frisches Ekzem an Fersen und Unterschenkeln. Stuhl vermehrt, dünn.

Verlauf: Wundsein gebessert. Ganz gute Zunahme bei Eiweißmilch. Kein foor. 2. XII. entlassen.

Xahrung 19. XI. bis 2. XII, 1912: Eiweißmilch, Tee, I. II. mit Schleim und $3-5^{\circ}$ o Nährzucker. Am 27. XII.: Eiweißmilch: 200 g., Tee: 40 g., I. II.: 440 g. mit Schleim und 5\% Nährzucker. 26. XII.: Eiweißmilch: $550 \mathrm{~g}$., Tee: $100 \mathrm{~g}$.

Gewicht: 27. XII.: 2850 g.; 26. XII.: 2870 g. Temperatur: 27. XII.: $36,8^{\circ}$; 26. XII.: $36,8^{\circ}$. Stühle: 27. XII. : $1 ; 26$. XII. 4 .

Die Untersuchung des Blutserums am 27.26. XI. ergibt:

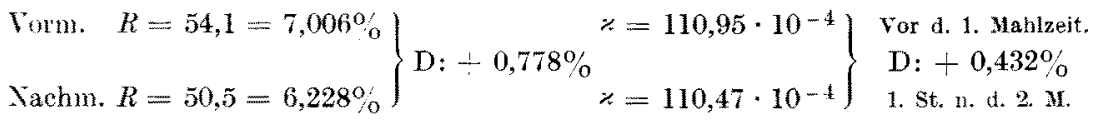

In diesem Falle ist der Wert für die Leitfähigkeit entschieden zu hoch. Der refraktometrische Wert zeigt eine unbeträchtliche Schwankung, während die Leitfähigkeịt in ihren Werten konstant bleibt.

Fall 8. Josef R., geb. 28. XI. 1912. Eintritt: 17. XII.; untersucht: 20./19. XII.

Alter: 3 Wochen. Gewicht: $2980 \mathrm{~g}$. am 17. XII. Diagnose: Dyspepsie. 
Anamnese: Pflegekind, keine Brust. Angeblich in jeder Windel Stuhl. Trinkt nicht.

Status: Sehr vernachlässigtes Kind mit frischem Ekzem im Gesicht. FuBsohlen wund, leicht blutend. Ekzem an den Unterschenkeln. Wund am Gesäß. Mundhöhle gerötet. Rachen frei. Herz und Lungen: 0 . B. Bauchdecken gut gespannt. Ernährungszustand leidlich.

Verlauf: 19. XII. Eiweißmilch wegen starker Dyspepsic - 23. XII. Eiweißmilch-Stuhl. Keine Zunahme. Allgemeinbefinden nicht gebessert.

Befund: Pirquet negativ.

Nahrung: 17. XII. bis 20. XII. 1912: I. II. mit Schleim und 5\% Xährzucker, Tee, EiweiBmilch (Schwankung in der Gewichtskurve!), Frauenmilch. Am 20. XII.: Eiweißmilch: $400 \mathrm{~g}$. mit 2\% Nährzucker, Tee: $175 \mathrm{~g},-19$. XI1.: I. II.: 125 g., Eiweißmilch: 150 g. mit $1 \%$ Nährzucker, Tee: 275 g.

Gewicht: 20. XII.: $2890 \mathrm{~g} . ; 19$. XII.: $2870 \mathrm{~g}$.

Temperatur: , " : $36,6^{\circ} ;, \quad, \quad: 36,6^{\circ}$.

Stühle: $\quad, \quad: 2 ;,, \quad, \quad$ : 4 .

Die Untersuchung des Blutserums am 20./19. XII. ergibt:

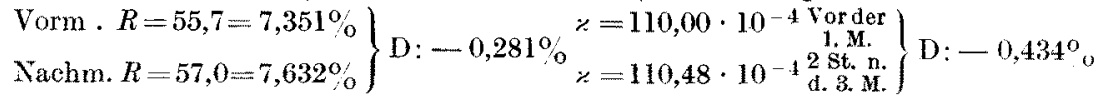

Der Säugling zeigt für sein Alter eine relativ hochgestellte Blutkonzentration mit Vermehrung der dissoziablen Salze, Nach der Resorption ist der Eiweißgehalt etwas größer, vielleicht ist diese Tatsache auf einen kleinen Wasserverlust oder, was wahrscheinlicher erscheint, auf mangelhafte Wasseraufnahme zurückzufïhren. DaB in diesem sehr jugendlichen Alter (3 Wochen) in Anbetracht der Drspepsie keine Schwankungen in den physikalisohen Konstanten vorkommen, läßt auf eine recht gute Konstitution dieses Organismus, auf eine vermehrte Resistenz schließen.

Fall 9. Karl Sch, geb. 10. VII. 1912. Eintritt: 19. X. und 6. XII. 1912; untersucht: 18. u. 20. XII. 1912.

Alter: 5 Monate. Gewicht: $3800 \mathrm{~g}$. Diagnose: Dyspepsie.

Anamnese mangelhaft. Die Mutter liegt schwer krank in der Frauenklinik. Pflegekind. Seit 3./4. X. sind die Stühle grün, dünn, gehäuft. Seit heute EYbrechen, nimmt keine Flasche mehr. Bekam Tee mit Milch.

Status: Sehr blasses, elend aussehendes, vernachlässigtes Kind. An den Beinen massenhafte, am Kopf und Rumpf vereinzelte, meist ganz verheilte Restc von Furunkeln und Pusteln. Turgor herabgesetzt, Haut bleibt etwas in Falten. Ernährungszustand stark reduziert. Sensorium frei. Bauchdeckenspannung ganz gut. Rachen und Lungen frei. Cor: o. B. Leber am Rippenbogen; Milz nicht fühlbar. An Hals und in der Axilla kleinste Drüsen. Stühle wässerig.

Ord.: Tee, Eiweißmilch.

Verla uf: 20. X. Fiebert, bricht, Stühle schlecht. - 21. X. Nimmt zu, Stühle besser, bricht nicht mehr. Temperatur normal. - 24. X. Stühle bei $4^{\prime \prime}$ Zucker zur FiweiBmilch wieder sehr schlecht, daher wird auf $3 \%$ zurückgegangen. - 
25. X. Fiebert. Gewicht hält sich, Stühle zum Teil gut, zum Teil schlecht. Hustet. Auf den Lungen kaum etwas hörbar, hier und da ein grobes Geräusch. Rachen: o. B. Fiechtes Trommelfell gerötet (?). - 26. X. Deutliche Besserung. Hautturgor bedeutend besser; Haut gut durchfeuchtet. Falten bleiben kaum mehr stehen. Ist noch recht blaß, lächelt. Temperatur geht herab. -28 . X. Hoher Tomperaturanstieg. Ursache?. Stühle leidlich. Sieht jedoch recht matt und blal aus, bricht oft. Falten bleiben wieder deutlich stehen. Daher Frauenmilch. 29. X. Temperatur von pyämischem Charakter. Hustet, auf den Lungen nur rechts hinten und neben der Wirbelsäule bei tiefen temzügen etwas Rasseln. Urin: o. B. Am Hinterhaupt tin kleiner Substanzdefekt (R. St eines Furunkels) mit gerötetem Kopf, kaum Kekretion. Ohren: o. B. Rachen und Nase: o. B. 30. X. Fieberfrei, entzündete Stelle am Hinterkopf frei. - 4. XI. Erholt sich bei Frauenmileh und $1 / 3$ Milch ohne Zucker, seit 3. XI. mit $2 \%$ Zucker. Doch noch recht blaB. -8 . XI. Sieht etwas besser aus. Das Gewicht hebt sich. Stühle zwischendurch verschlechtert, jetzt wieder besser. - 15. XI. Nimmt zu. Stühle gut. - 22. XI. Gewicht: 4000 g. - 3. XII. Gewicht: 3820 g. Temperatur: $37,7^{\circ}$, Seit 3 Tagen krampfhafter Husten; rechts hinten unten klingendes Rasseln. Thera pie: Codein 0,01 $2 \mathrm{mal} \mathrm{tägl.;} 2 \mathrm{mal}$ Bad mit GuB. - 6. XII. Hustet stärker, stuhl geregelt, sehr elendes Aussehen.

Nahrung 19. X. bis 6. XII. 1912: Tee, Eiweißmilch mit 2\% Nährzucker, Ringersche Lösung, Frauenmilch, Buttermileh, I. I. mit Schleim und $5 \%$ Nährzucker, $3 \%$ Haferschlein.

Wiederaufnahme:

Status: 6 . NII. Sehr viel schlechter als bei der Entlassung. Blasses, elendes Kind. Haut welk, Muskulatur schlaff. Fettpolster fast völlig geschwunden. Bauchdecken weich. Leber fühlbar. Tensorium frei. Lunge: besonders über der rechten Lunge reichliches Rasseln.

Verlauf: 7 . XII. Beiderseits hinten reichlich klingendes Rasseln. Hat sich schon etwas erholt. Stuhl leidlich. Ord.: Frauenmilch. Bad mit Überguß. 11. XII. Auf den Lungen kaum mehr etwas zu hören. Hustet noch viel (Pertussis?). Erholt sich sonst. - 13. XII. Hustet weniger. Lungen fast frei. - 18. XII. Lungen frei, nimmt zu. - 20. XII. Entlassen.

Thera pic: Warme Ohrumschlage mit essigsaurer Tonerde; $2 \mathrm{mal}$ Bad mit ẗbergub.

Befund: Pirquet negativ.

Nahrung: 6. XII. bis 20. XII. 1912: Tee, Frauenmilch, I. II. mit Sichleim und $5 \%$ Nährzucker, I. I. mit Schleim und $5 \%$ Nährzucker. Am 18. XII.: I. I.: 800 g.; 20. XII.: I. I.: 420 g.

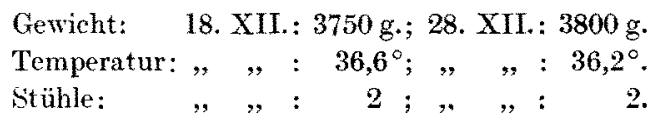

Die Untersuchung des Blutserums ergibt:

18. XII.:

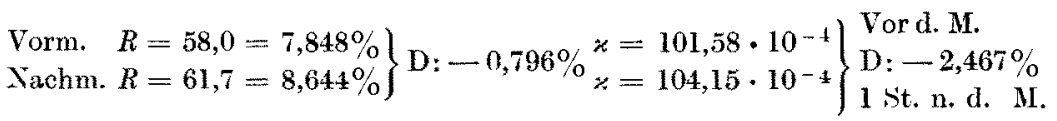


20. XII.:

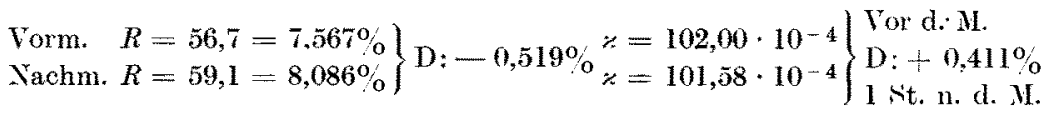

Beide Untersuchungen zeigen eine stärkere Eindickung des Blutes mit normalem Verhalten der dissoziabeln Substanz. Das Kind hat nur I. I. als Nahrung erhalten zurZeit der Untersuchungen. KleinereSchwankungen, bedingt durch die Resorption, kommen vor, dabei zeigen Leitfähigkeit und Refraktometerwert ein refraktäres Verhalten; die Ausschläge bewegen sich jedoch in der normalen Variationsbreite. Das eigentümliche Verhalten des Organismus berechtigt zu der Annahme, daB zur Zeit der Resorption ein Wasserverlust mit minimalem Verlust. ar dissoziabler Substanz statthat.

Fall 10. Margarete F., geb. 25. VII. 1912. Eintritt: 1. X., 12. XI., 28. XI.; untersucht: 13. XII. 1912.

Alter: $4 \frac{1}{2}$ Monate. Diagnose: Dyspepsie, Pneumonie.

1. X. Eltern gesund. Unter 5 Geburten 2 Frühgeburten. Pat. wurde ausgetragen. 8 Wochen gestillt, seitdem nur 1-2 mal gestillt, dazu l' Milch und 4' 'Thleim. Seit 3 Tagen Erbrechen; dazu seit 8 Tagen Durchfall. Daher EiweiB mit Zucker. Husten seit 3 Wochen.

Status: Sehr elendes Kind. Schlechter Hautturgor, Haut wenig elastisch. Ciesichtsfarbe graublaß. Fast keine Muskulatur. Keine Fettpolster. Große Fontanelle sehr weit, etwas eingesunken. Starkes schwarzes Kopthaar. Intertrigo am GesäB. Einzelne Drüsen am Hals fühlbar. Nicht benommen, ganz lebhaft. Lungen und Herz: o. B. Bauch leicht aufgetrieben. Stuhl schlecht. Milz und Leber nicht palpabel. Hals gerötet. Schleimhäute blaß.

Verla uf: 4. X. Aussehen besser. Trinkt ordentlich. Stüle ganz gut (EiweiBmilchstühle). - 7. X. Aussehen .mäBig. - 11. X. Absolut keine Zunahme. Daher auf $1 / 3$ Schleim gesetzt. Gewióht: $3130 \mathrm{~g}$. - 15. X. Bei leidlichem Befinden mit geringer Gewichtszunahme entlassen.

Wiedera uf nah me: 12. XI. Seit 3 Tagen Durchfälle. Mageres, sehr zurückgebliebenes Kind. Bauch etwas aufgetrieben. Stuhl dünn, grün, vermehrte Zahl. Hautfarbe besser als 1 . X. Stark wund am Gesäß. Mund etwas gerötet. Lunge und Herz o. B. Leber und Milz nicht palpabel.

Verlauf: 14. XI. Bei Eiweißmileh Abnahme. Stühle fest, hell. - 15. XI. Zunahme. Eiweißmilchstuhl. Gute Stimmung. - 21. XI. Davernd gute Zunahme. Stuhl o. B. Allgemeinbefinden gut. - 22. XI. Umgesetzt auf I. I. Gewicht: $3570 \mathrm{~g}$.

Wiedera ufnahme: $28>$ XI. Gewicht: $3570 \mathrm{~g}$. Hustet seit 4 Tagen. Mageres Kind. Blasse Farbe. Atmung beschleunigt. Ppastische Hustenanfälle. Mund sauber. Lungen: rechts hinten unten viel feines Rasseln. Bauch: o. B.

Verla uf: 5. XII. Rechts hinten unten feines Rasseln. Rechts vorn unten Bronchialatmen und Rasseln; rechts hinten oben Bronchophonie, leichte Dämpfung. - 8. XII. Seit gestern entfiebert. - 13. XII. Wenig Husten, kein Lungenbefund. 
Xahrung 28. XI. bis 13. XII. 1912.: I. I. mit Schleim und 5\% Nährzucker: $8 ; 0 \mathrm{~g}$.

Gewicht: 12. XII.: 3380 g.; 13. XII.: 3340 g. ; 14. XII.: 3420 g. Temperatur: 13. XII.: $35,8-36,8^{\circ}$.

Die Untersuchung des Blutserums am 13. XII. ergibt:

$\left.\left.\begin{array}{ll}\text { Vorm. } & R=58,1=7,870 \% \\ \text { Nachm. } R=54,6=7,114 \%\end{array}\right\} \mathrm{D} ;+0,756 \% \begin{array}{l}\varkappa=102,00 \cdot 10^{-4} \\ \varkappa=105,03 \cdot 10^{-4}\end{array}\right\} \begin{aligned} & \text { Vor d. 1. M. } \\ & \text { D: -2,884\% } \\ & \text { Nach d. } 2 . \text { M. }\end{aligned}$

Der hohe Eiweißwert mit zugehöriger kleiner Tagesschwankung weist hier auf einen geringen Wasserverlust hin. Es besteht weiter eine normale Leitfähigkeit mit refraktärem Ausschlag innerhalb normaler Grenzen. Es spielt offenbar die früher vorhanden gewesene Dyspepsie bei dieser Blutkonzentrationserhöhung noch zum Teil mit.

Fall 11. Rosa R., geb. 18. IX. 1912. Eintritt: 30. XII.; untersucht: 31./30. XII. 1912. u. 10./13. I. 1913.

Alter: $3 \frac{1}{2}$ Monate. Diagnose: Scabies, Dyspepsie.

An amnese: Noch 4 Kinder. Alle haben einen Ausschlag. Pat. wurde ausgetragen, spontan geboren; nicht gestillt. Hat $4^{\prime}$ Schleim $+4^{\prime}$ Milch 2stündl., nachts auch. Ausschlag seit 4 Tagen. Stuhl gut.

Status: Sicabiesefflorescenzen, zum Teil zerkratzt. Leidlich genährt. Zunge sauber, Rachen frei. Lunge und Herz: o. B. Bauch: o. B.

Verlauf: 1. I. 1913. Besserung der Scabies. - 4. I. Besserung des Ausschlags vorhanden. - 7. I. Dünne Stühle. Gewichtsabnahme. 2 Teemahlzeiten. Ċbergang auf $1 / 3$ Mileh mit Hafersehleim ohne Zucker. - 9. I. Stühle besser. $10 \mathrm{~g}$. Nährzucker zugelegt. - 14. I. Stuhl in Ordnung. Scabies geheilt. Impetiginöses Ekzem auf dem behaarten Kopf. - 21. I. Frei von Ekzem und Scabies entlassen.

Befund: $30 . / 31$. XII. 1912. Gewicht: $5610-5480 \mathrm{~g}$. 10. I. 1913 . Gewicht: 5250 g.; 11. I.; 5220 g.; 12 . I.: 5280 g.; 13. L.: 5270 g. Stühle 31./30.: je 1. Temperat.: 31. XII.: $37,2^{\circ}$; 30. XII.: $36,4^{\circ}-37,4^{\circ}$. - Stühle 10./13. I.: je 1. Temperat.: 10./13. I.: je $37^{\circ}$.

Nahrung: 30. XII. bis 31. XII. 1912.: Tee I. II. mit Schleim und $5 \%$ Nährzucker, I. I. mit s̀chleim und 5\% Nährzucker. - Am 31. XII.: I. II. : 300 g.; I. I.: 450 g. m. $5 \%$ Nährz.; 30 . XII.: I. II. : 300 g. m. Schleim u. $5 \%$ Nährz.; Tee: 120 g. - 10. I.: I. II.: 750 g. m. 20 g. Nährz.; I. I. m. Sohleim u. $3 \%$ Nährz. - 13. I.: I. I.: 300 g.; I. II.: 450 g.; I. I. m. Schleim u. $5 \%$ Nährz.

Therapie: Schwefelbad. Bals. peruv. Verb. mit Lenigallol-Zinkpaste.

Die Untersuchung des Blutserums ergibt:

31./30. XII.:

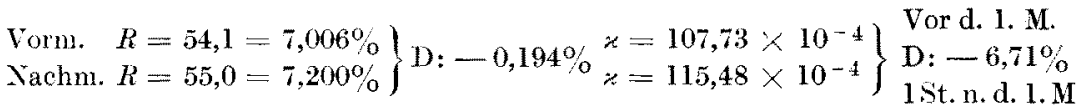

10./13. I. 1913 :

$\left.\begin{array}{l}\text { Vorm. } \quad R=55,6=7,329 \% \\ \text { Nachm. } R=54,5=7,092 \%\end{array}\right\}$ D: $\left.+0,237 \% \begin{array}{c}x=103,73 \\ *=103,73\end{array}\right\}$ D: $0 \begin{aligned} & \text { Vor d. 1. M. } \\ & 1 \text { St. n. d. 2. M. }\end{aligned}$ 
Vor allem fällt der große Ausschlag der Werte für die Leitfähigkeit am 31. XII. 1912 auf, der bedingt ist durch Wasserverlust, wie aus dem Gewichtssturz deutlich erhellt. Das refraktäre Verhalten ist ebenfalls nach der Gewichtskurve leicht zu deuten, da der Wasserverlust vom 30. auf den 31. XII. statthat. $R$ und $\psi$ nachmittags wurden am 30. XII. schon untersucht. Trotz des gewaltigen Wasserverlustes ist die Leitfähigkeit recht hoch; so wäre anzunehmen, daß kein nemnenswerter Salzverlust mit der Wasserabgabe eingetreten ist. Der Eiweißgehalt ist etwas zu hoch, eine Tagesschwankung ist jedoch nicht zu verzeichnen. Die Untersuchungen rom 10. I. 1913 zeigen im Eiweißgehalt und besonders in der Leitfähigkeit normale Verhältnisse, $d . h$. es tritt uns hier' keine Tagesschwankung mehr entgegen.

Interessant ist bei diesen Fällen von Dyspepsie die relativ hohe Blutkonzentration, ohne daß ein nennenswerter Verlust an dissoziabler Substanz zu verzeichnen wäre.

Fall 12. Erhard N., geb. 8. IX. 1912. Eintritt; 2. X. u. 16. XI. 1912; untersucht: 21. XI.

Alter: $2^{1 / 2}$ Monate. Gewicht: 3140 g. am 2. X. Diagnose: Dyspepsie.

Anamnese: Ausgetragen, spontan geboren. Gestillt 5 Wochen. Dam 3) Teile Wasser und 2 Teile Milch + Vilchzueker alle $21 / 25$ tunden. Nachts $1-2$ mal: 7-8 Flaschen. Dabei mager geworden, Stuhl 6-8 mal. dünn. Darauf 3 Teilo sichleim +2 Teile Mileh + Milchzucker: $7-8$ Flaschen. Dann 1 Tag Nestle $\div$ Milch. Am 30. IX. beim Arzt: Tee (mit Zucker) verordnet; seither nur Tee, seit gestern mit Saccharin. Stuhl grünbraun.

Status: Kleines, mageres und blasses Kind. Schädel etwas groß. Fontanelle leicht eingesunken! Turgor leidlich, Fettpolster gering. Bauchdeckenspannung noch ganz gut. Lunge und Cor: 0 . B. Kleinste Drüsen.

Therapie: Ord.: 300 Eiweißmilch $+3_{\%}^{\circ}$ Nährzucker.

Verlauf: 9. X. Sieht ganz gut aus, nimmt zu; Stühle gut. - 5. X. Auf $1 / 3$ Milch umgesetzt. Geht gut. -7 . X. Sieht recht gut aus, ist ganz rund geworden.

Wiedera uf nahme: 16. XI. 3912 . Gewicht: $3340 \mathrm{~g}$. Soll dauernd erbrechen und bis 6 Stühle haben. Blaß, mager, reduziert. Lungen und Herz frei.

Nahrung: 2. X. bis 7. X. 1912: Eiweißmilch + Tee + I. II. mit Schleim und $5 \%$ Nährzucker.

Wiedera ufnahme. Xahrung: 16. XI. bis 20. XI. 1912.: Frauenmilch, Tee, 1. II. mit Sichleim und $5 \%$ Nährzucker, Buttermilch, 1. II. mit Schleim und $5 \%$ Nährzueker.

Befund: Pirquet negativ.

Die Untersuchung des Blutserums ergibt:

21. XI.:

$\left.\left.\begin{array}{l}\text { Vorm. } R=50,0=6,120 \% \\ \text { Nachm. } R=51,7=6,487 \%\end{array}\right\} \mathrm{D}:-0,365 \% \begin{array}{l}\varkappa=109,00 \times 10^{-1} \\ \%=118,30 \times 10^{-4}\end{array}\right\} \begin{aligned} & \text { Vord. Mahlzeit } \\ & \mathrm{D}:-7,861 \% \\ & 1 \text { St. n. d. M. }\end{aligned}$ 
Ein weiterer Fall von Dyspepsie durch Uberfütterung liegt hier vor. Der refraktometrische Wert ist normal mit irrelevanter Schwankung an diesem Tage. Die Leitfähigkeit erreicht recht beträchtliche Werte und weist eine ansehnliche Schwankung auf, und zwar zeigt sie ein refraktäres Verhalten. Die Nahrung besteht an diesem Tage aus: Abgedr. Fr. M.: 300 g., I. II. : 300 g., B. M. : 100 g., Tee: 50 g. "Es bestand weiter eine Gewichtszunahme von $20 \mathrm{~g}$. Gewicht: $3420 \mathrm{~g}$. am 21. XI.; Stühle: 3 ; Temperatur: $36,4^{\circ}$.

Fall 13. Elsa H, geb, 19. VIII. 1912. Eintritt: 31. XII.; untersucht: 3./13. I. und 22. I. 1913.

Alter; 5 Monate. Diagnose: Mehlnährschaden.

Anamnese: Erstes Kind, ausgetragen, spontan geboren, Brust 3 Wochen, dann Vollmilch 6 Wochen, darauf Durchfall und Erbrechen. In ärztlicher Behandlung. 14 Tage nur Tee, hernach 7 Wochen Schleim. Seit 8 Tagen etwas Milch. Trinkt schlecht. Stuhl düm, schreit beim Wasserlassen.

Status: Elendes, sehr blasses Kind. Haut schlaff. Die Falten gleichen sich jedoch schnell wieder aus. Ernährungszustand schlecht. Hypertonie der Glieder. Lungen: hinten unten etwas Rasseln. Cor: o. B. Milz nicht, Leber gut fühlbar.

Verlauf: 2. I. Hustet viel, reichl. Rasseln auf den Lungen. - 6. I. Klingendes Rasseln: Ôdeme. Fieber. Rechts hinten unten neben der Wirbelsäule ein etwa fünfmarkstückgroßer Bezirk mit Bronehophonie, bronehialem Hauch und klingendem Rasseln. Ödeme. - 8. I. Fieberfrei. Pneumonischer Herd rechts unten unverändert. Pirquet negativ. Ödeme besser. Sonst kein Befund.

Nahrung: 3. I. Abgedrükte Franenmilch: $500 \mathrm{~g}$. Tee: $250 \mathrm{~g}$. ; Gewicht: $3900 \mathrm{~g}$; Stühle: 2 ; Temperatur: $37,2^{\circ}$ am 3. I. - 13. I. Abgedrückte Frauenmilch: 500 g., I. II. 200 g., Tee: 100 g.; Gewicht: 3920 g.; Stühle: 4; Temperatur: $37,6^{\circ}$ bis $36,8^{\circ}$ am 13 . I.

22. I.: Abgedrückte Frauenmilch: 300 g.; I. II.: 400 g.; I. I.: 100 g. mit $5 \%$ Nährzucker. Gewicht: 4100 g.: Stühle: 2 ; Temperatur: $36,6^{\circ}$ bis $36,8^{\circ}$.

Die Untersuchung des Blutserums ergibt:

3. 13. I.:

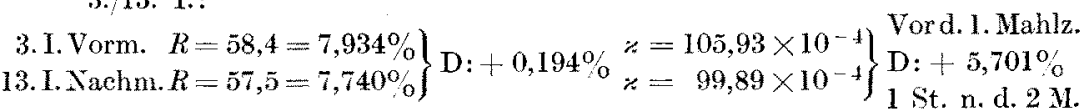

22. I. :

$\left.\begin{array}{l}\text { Vorm. } \quad R=57,6=7,762 \% \\ \text { Nachm. } R=57,4=7,718 \%\end{array}\right\}$ D: $\left.+0,044 \% \%=101,15 \times 10^{-4}\right) \begin{aligned} & \text { Vor d. 1. M. } \\ & \text { D: }-0,833 \% \\ & \text { Nach d. } 2 . \text { M. }\end{aligned}$

Bei dem 5 Monate alten Kinde ist der hohe Eiweißwert auffallend, der in einer erhöhten Blutkonzentration begründet sein dürfte. Wir haben hier ein Kind vor uns, das einen schweren Mehlnährschaden sich zugezogen hatte, damit dürfte der große Ausschlag in der Leit. fähigkeit in Zusammenhang $z u$ bringen sein. Am 3./13. I. hat eine größere Schwankung in der Leitfähigkeit statt, die am 22. I. nicht 
mehr zu verzeichnen ist. Die Tageswerte der lichtbrechenden Substanzen sind annähernd konstant. Der betreffende kindliche Organismus verfügt in Anbetracht der schweren Schädigung offenbar über eine entsprechend gute Konstitution ${ }^{1}$ ).

Fall 14. Paula M., geb. 9. IX. 1912. Eintritt: 22. XI.; untersucht: 7./6. und 12. XII. 1912.

Alter: 3 Monate. Geb.-Gewicht: 5000 g. Diagnose: Chron. Ernährungsstörung.

A namnese: Ein Kind $2^{1 / 2}$ Jahre alt. Zwillingskind (Zwilling gestorben), spontan geboren, ausgetragen, Mutter lungenkrank, stillt deshalb nicht. Nachts 1 mal die Flasche, sonst 2 stündlich. Jedesmal 4 Teile Milch und 4 Teile Schleim. Stuhl 5-6 mal am Tage. Ganz wenig Zucker. Schüttet sich aus, Stuhl weehselnd.

Status: Ganz nettes Kind, etwas mager und klein. Starker Intertrigo. Stuhl hier gut. Geringe Nabelhernie. Temperatur 35,9 $9^{\circ}$ Ord.: $1 / 3$ Milch 6 mal. Arg. Zinkpaste, Nabelpflaster.

A ufnahme: 22. XI. Gewicht: $2870 \mathrm{~g}$. Soll nach jedem Sichoppen brechen. bekommt $2^{\prime}$ Milch mit 6 ' Sohleim.

Status: Schlecht gepflegtes, schmutziges Kind. Stark nässender Intertrigo inguinalis bis auf die Unterschenkel sich fortsetzend. An der rechten Fußsohle Eiterblase. Mund sauber. Lunge, Herz, Bauch: o. B.

Verlauf: 30. XI. Sehr schlechte Stühle. - 9. XII. Immer noch recht jämmerlich. - 17. XII. Weitere Abnahme. Schlechte Stühle.

Thera pie: Arg. nitric., Incision. Lenigallol-Zinkpaste, Wärmflaschen, Darm. spülung.

Nahrung: I. II. mit Schleim und 5\% Nährzucker, Frauenmilch, Tee, $3{ }^{\circ}$ Haferschleim ohne Zucker, I. I. mit Schleim und $5 \%$ Nährzucker. Am 7 . XII.: Frauenmilch, abg.: 200 g., I. II. mit Schleim und $5 \%$ Nährzucker: $460 \mathrm{~g}$. 6. XII.: abg. : 200 g., I. II. mit Schleim und $5 \%$ Nährzucker: 400 g. - 12. XIl.: abg.: $400 \mathrm{~g}$. Frauenmilch, I. II.: $300 \mathrm{~g}$. ohne Zucker.

Gewicht: 7. XII.: 2730 g.; 6. XII.: 2760 g.; 12. XII.: 2680 g. Temperatur: 7. XII.: $36^{\circ}-37^{\circ} ; 6$. XII.: $36,5^{\circ}-35,4^{\circ}-37^{\circ} ; 12$. XII.: $36,2^{\circ}-37^{\circ}$. Stühle: 7.6. XII.: je 1; 12. XII.: 5. NB. 2 Wärmeflaschen am 6. und 7. XII. 1912.

Befund: Pirquet negativ.

Die Untersuchung des Blutserums ergibt folgendes Resultat:

7. XII.

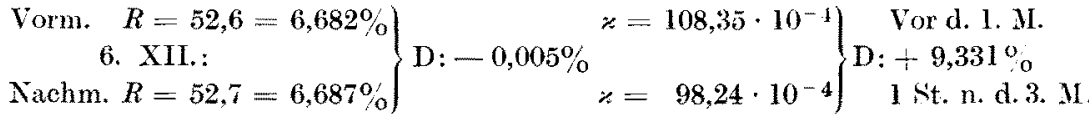
12. XII.:

$\left.\left.\begin{array}{r}\text { Vorm. } R=54,6=7,114 \% \\ \text { Nachm. } R=50,7=6,271 \%\end{array}\right\} \begin{array}{r}\varkappa=92,75 \cdot 10^{-1} \\ \mathrm{D}:+0,843 \% \\ \%=101,58 \cdot 10^{-1}\end{array}\right\} \begin{gathered}\text { Vor d. } 1 . \mathrm{M} . \\ \mathrm{D}:-8,692 \% \\ 1 \text { St. n. d. 2. M. }\end{gathered}$

Der Eiweißgehalt vom 7. XII. ist normal und ohne Schwankungserscheinungen. Die dazugehörige Leitfähigkeit zeigt vormittags einen

1) Vgl. u. a. auch B. Salge, Ein Beitrag zur Pathologie des Mehlnährschadens der Säuglinge. 
recht hochgestellten Wert, nachmittags dagegen sinkt die Leitfähigkeit unter die normalen Grenzwerte weit hinab. Der betreffende Organismus besitzt eine recht schlechte Regulierungsfähigkeit der physikalischen Zusammensetzung seines Blutserums. 7. XII. 1912: Gewicht: $2760 \mathrm{~g}$.: Nahrung: Abgedrückte Frauenmilch: 200 g.; 6. XII. : Gewicht: 2730 g.; Nahrung: I. II. : $400 \mathrm{~g}$.

Das Ergebnis der zweiten Untersuchung zeigt ebenfalls ganz erhebliche Aussehläge sowohl in den Eiweißwerten als auch in dem jetzt refraktären Wert der Leitfähigkeit. Bei einem Vergleich dieser Daten mit der Krankengeschichte stellt es sich heraus, claß für diese großen Schwan kungen ein Wasserverlust durch vermehrte Stühle (11. XII: 4, 12. XII: 5, 13. XII : 6; Nahrung: 12. XII: Abgedrückte Franenmilch: 400 g., I. II. : $300 \mathrm{~g}$.) anzuschuldigen ist, zumal der dem Wasserverlust parallel gehende enorme Gewichtssturz (11. XII.Gewicht :2710g.. 12. XII:2680g., 13. XII.: 2650 g.) im Verein mit dem jämmerlichen Allgemeinbefinden des Kindes diese Auffassung vollauf bestätigt. Am 12. XII. vormittags tritt eine kleine Bluteindickung zutage mit sichtlicher Verminderung der für die Leitfähigkeit in Betracht kommenden Salze. Nach der Resorption stellen sich beiderseits die normalen Werte wieder ein.

Dieser Organismus besitzt ein ganz schlechtes Vermögen, seine Blutkonzentration im Verlaufe des Tages ausreichend zu regulieren. Dieser Fall liefert abermals einen Beitrag zur Begründung der Erfahrungstatsache. daß die alimentären Störungen um so erschwerender für das Wohl und Wehe des Kindes ins Gewicht fallen, je jünger der Säugling ist. Auch wurde das Find zudem noch von Anfang an mit einer artfremden Nahrung aufgezogen, was es ja bekanntlich von vornherein eine ungünstigere Stellung im Lebenskampfe einnehmen läßt.

Fall 15. Alfred M., geb. 16. VIII. 1912. Fintritt: 7. I. 1913; untersucht: 9./13. I., 17. 1. u. 23 . I. 13 .

Alter: 5 Monate. Diagnose: Spasmophilie.

Pflegekind, gestillt? Bekam Schleim und Milch. Seit 4 Wochen $4^{\prime}$ Milch $+4^{\prime}$ Schleim 3 stdl., nachts 3 stdl. Stuhl in jeder Windel. Zieht beim Schreien, wird blau, sehr oft, seit 4 Wochen.

Status: In der Entwicklung anscheinend etwas zurückgebliebenes Kind. Ernährungszustand gut. Sehr blaß, schlaff. Dicker Bauch. Starke Kraniotabes. Geringer Rosenkranz. Lunge: etwas grobe Geräusche. Cor.: 0 . B. Leber: 1 Finger unter dem Rippenbogen. Milz: nicht fühlbar. Facialisphänomen schwach positiv (Hirnast). Zieht etwas beim Schreien.

Verlauf: 9. I. KÖZ. 4 MA. Zieht hier und da. Facialisphänomen rechts positiv, Trousseau positiv. -16 . I. KÖZ. über $5 \mathrm{MA}$. Zieht nicht mehr.

Pirquet: negativ. 
9. I. KÖZ. 4 MA. Gewicht: 5190 g. - 13. I.: 5130 g. Nahrung: I. II.: 1000 g. Stühle: 3. - 13. I. Temperatur: $37^{\circ}$. Stuhl: 1. -- 17. I. Gewicht: $\mathbf{3 2 6 0 \mathrm { g } .}$ Nahrung: I. II.: 800 g., I. I.: 200 g. I. II.: 1000 g. am 13. I. bis 17. I. Ntühle: 2. - 22. I. Gewicht: $\mathbf{5 2 7 0}$ g., 23. I. Gewicht: $\mathbf{5 3 6 0}$ g. Nahrung: I. I.: $600 \mathrm{gg}$, I. II.; $200 \mathrm{~g}$., Suppe: $200 \mathrm{~g}$.

Die Untersuchung des Blutserums ergibt:

9./13. I.:

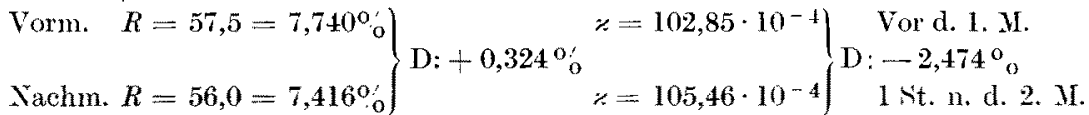
17. I.:

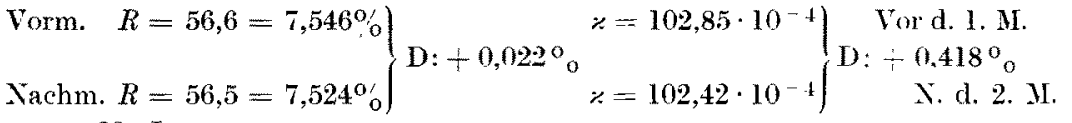
23. I.:

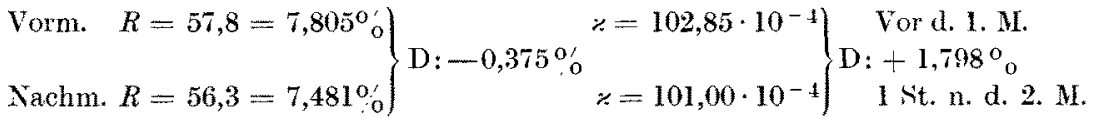

Die Spasmophilie gehört bekanntlich als krankhafter Zustand des Nervensystems zu den durch eine Stoffwechselstörung hervorgerufenen Krankheiten. Interessant ist in diesem Falle die Tatsache, daß die am 9. I. rorkommende. immerhin kleine Tagesschwankung der Werte für den Eiweißgehalt und die Leitfähigkeit mit der Besserung des Krankheitsbildes einem normalen konstanten Verhalten am 17. I. Platz macht.

Die Eiweißwerte sind durchstehend etwas hoch; die Leitfähigkeit hingegen weist bei allen $\mathrm{C}^{\top}$ ntersuchungen normale Zahlen auf.

Fall 16. Frieda T., geb. 25. IX. 1912. Eintritt: 25. XI.: untersucht: 7, XII. Alter: $2^{1 / 2}$ Monate. Diagnose: Lues.

A nam nese: 4 Stiefgeschwister gesund. Augenklinik: Irisprolaps. 4 Wochen zu früh geboren, keine Fehlgeburt. Schnupfen, Milztumor. Soll auch einen Aus. schlag kurz nach der Geburt gehabt haben. Hat $1 / 3$ Milch erhalten.

Status: Kleines, schlecht genährtes Kind. Schnupfen. Mund sauber, Rachen frei. Irisprolaps am linken Auge. Lungen und Herz: o. B. Milz und Leber deut. lich palpabel. Stuhl schleimig.

Verlauf: 30. XI. Wassermann positiv. Gesicht nimmt gelblich-fahle Farbe an. - 4. XII. Stuhl besser. - 11. XII. Stuhl geregelt. Befinden ungeändert. (iewicht am 7. XII.: $2530 \mathrm{~g}$. Kleine Schwankungen vorher und nachher um $2500 \mathrm{~g}$.

Nahrung: Frauenmilch: 400 g., Tee, I. II. mit Schleim und $5 \%$ Nährzucker: $200 \mathrm{~g}$.

Gewicht: 6. XII. 2500 g., 7. XII.: 2530 g. St ühle: 2 . T emperatur: $36,8^{\circ}$ bis $37,0^{\circ}$.

Die Cntersuchung des Blutserums am 7. XII. ergibt:

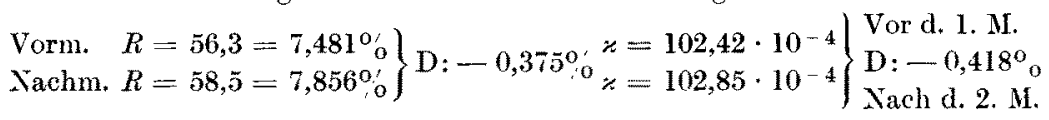


Der zu hohe Eiweißwert des Kindes kommt dem des Erwachsenen nahe. Die Leitfähigkeit ist gut. Die Tagesschwankungen des Refraktometer - wie auch des Leitfähigkeitswertes sind irrelevant.

Fall 17. Berthold P., geb. 2. IX. 1912. Eintritt: 16. XII.; untersucht: 18. XII. Alter: $3^{1 / 2}$ Monate. Gewicht: $5100 \mathrm{~g}$. Diagnose: Rachitis.

Anamnese: Pflegekind. Brust 8 Wochen hindurch, dann $1 / 2$ Milch. Schreit seit 8 Tagen, zieht, schreckt zusammen, hustet wenig, Stuhl geregelt, palpable Milz.

St atus: Kräftiges, gut genährtes Kind, fieberfrei. Lunge und Herz: o. B. Milz nicht palpabel. Leber 1 Finger unter dem Rippenbogen. Deutliche Kraniotabes, Rosenkranz. Nägel an 3 Fingern der Hand verkümmert. Kein Facialisphänomen.

Verlauf: 18. XII. Wassermann negativ. Ist ruhig. Temperatur normal.

Nahrung: I. I. mit Schleim und $5^{0} ;$ Nährzucker: $900 \mathrm{~g}$.

Gewicht: 17. XII.: 5000 g., 18. XII.: 5100 g. Stühle: 18. XII.: 3, 17. XII.: 4. Temperatur: $36,8^{\circ}$ bis $37,0^{\circ}$.

Therapie: 3 Löffel Phosphor-Lebertran.

Die Untersuchung des Blutserums am 18. XIr. ergibt:

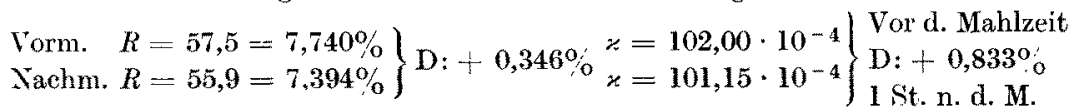

Trotz der vermutlichen Veränderungen im Salzstoffwechsel bei Rachitis haben wir hier für die Leitfähigkeit normale Werte. Die lichtbrechenden Substanzen zeigen einen etwas hohen Prozentgehalt. Schwankungen der beiden Tageswerte sind nicht zu verzeichnen.

Fall 18. Lina Sch., geb. 27. I. 1912. Eintritt: 18. XI.; untersucht: 29. XI.

Alter: 10 Monate. Diagnose: Impetigo contagiosa, Cystitis.

Anamnese: Pflegekind. Aufnahme wegen Impetigo contagiosa.

it atus: Dickes, gut genährtes Kind. Gesicht bedeckt mit Efflorescenzen von Impetigo contagiosa. Nackendrüsen geschwollen, Rachen frei. Innere Organe: o. B. Stuhl geregelt. Urin trüb, enthält viel Leukocyten.

Verla uf: 24. XI. Ausschlag gebessert. - 30. XI. Ausschlag geheilt. Mit Urotropin entlassen.

Nahrung: 29. XI.: I. I. : 800 g. mit Mehlsuppe und $5_{0}^{\circ}$ Nährzucker + Ge. mischte Kost: $170 \mathrm{~g}$.

Gewicht: 29. XI.: 8950 g.; 28. XI.: 8990 g. : 30. XI.: 9110 g. Temperatur: 37 . Stühle: 2 .

Untersucht am 29. XI.:

$\left.\begin{array}{rl}\text { Vorm. } R=54,2=7,027^{\circ} & \%=108,30 \cdot 10^{-4} \\ \text { Nachm. } R=54,8=7,157^{\circ},{ }_{o}\end{array}\right\} \begin{gathered}\text { Vor d. 1. M. } \\ \mathrm{D}:+2,437 \% \\ 1 \text { St. n. d. 2. M. }\end{gathered}$

Wir haben es hier mit einem sonst gesunden Kinde zu tun. Der Eiweißgehalt ist für sein Alter als normal zu bezeichnen. Die Leitfähigkeit zeigt relativ hohe Werte. 
Die Tagesschwankung der beiden Leitfähigkeitswerte ist zu geringfügig, um daraus weitere Schlüsse bezüglich der Blutkonzentration ziehen zu dürfen.

\section{III.}

Das Ergebnis dieser Untersuchungen ist somit dahin zusammenzufassen, daß der gesunde jugendliche Organismus des Säuglings unter normalen äußeren Bedingungen wohl imstande ist, die physikalische Zusammensetzung seiner Körpersäfte im Verlaufe des Tages konstant zu erhalten, was insonderheit die oben näherhin behandelten Fälle 1-10 und 15-18 deutlich lehren. Es kommen demnach irgendwie wesentliche Tagessehwankungen der Blutkonzentration, bezogen auf den Hungerzustand und die Resorption der Nahrung beim gesunden Kinde. das mit arteigener Nährflüssigkeit versehen wird, nicht vor, und die Ansicht, daß auch der gesunde Säugling selbst unter arteigenen Bedingungen erhebliche Schwankungen im Eiweißgehalt und in der Leitfähigkeit aufweise, die durch die tägliche Nahrungsaufnahme bedingt seien, besteht also schwerlich zu Recht. Soweit vermag der infantile Organismus die physikalische Zusammensetzung seiner Körpersäfte schon zu regulieren. Sobald jedoch eine Schädigung infolge einer Ernährungsstörung hinzutritt, so erhält dieser Regulationsvorgang cinen höchst labilen Charakter, der sich in größeren oder geringeren, durch die großen Wasserschwankungen des alimentärerkrankten Säuglingsorganismus bedingten Schwankungen der lichtbrechenden Substanzen und der Leitfähigkeit kundgibt, welche eben in dem Unvermögen des infantilen Organismus, seinen osmotischen Druck ${ }^{1}$ ) unter solchen Bedingungen konstant zu erhalten, ihre Erklärung haben. Dieses Verhalten auf Grund von Ernährungsstörungen zeigen deutlich die untersuchten Fälle: $11-14$ und 16 in ihren erheblichen Tagesschwankungen. Die größere oder weniger große, durch Ernährungsstörung verursachte Schä digung des Organismus und seiner Regulationsvorgänge hängt besonders von der guten oder schlechten Konstitution des einzelnen Individuums ab, wie zu Anfang sehon erwähnt wurde. Dabei kommt noch dem Alter²)

1) Vgl. die beigegebene Tabelle.

2) An dieser Stelle sei auch auf die interessanten Versuche an Hunden hin. gewiesen, die P. Schulz (Wachstum und osmotischer Druck bei jungen Hunder. Mitteil. I. u. II; Zeitschr. f. Kinderheilk. 3) im Anschluß an B. Salges Feststellungen über die Regulationsfähigkeit des osmotischen Druckes vorgenommen hat. Das Ergebnis faßt Schulz in folgenden Sätzen zusammen: ,Die Unvollkommenheit dieser Fähigkeit [der Konstanterhaltung der physikalischen Eigenschaften 
des betreffenden Säuglings eine ausschlaggebende Bedeutung für die Größe der Schädigung insofern $z u$, als der junge Säugling viel leichtêr eine dauernde Schädigung davonträgt, zum mindesten eine wesentlich längere Reparationszeit aufweist als das ältere Kind.

des Blutes] zeigt sich um so deutlicher, je länger, um so weṇiger, je kürzer die säuglingsperiode ist." In seiner ersten Mitteilung zieht er aus seinen Versuchen folgenden Sichluß: „Hunde jenseits der Säugungsperiode sind imstande, auch unter sehr ungünstigen Ernährungsbedingungen die physikalischen Eigenschaften ihres Blutserums innerhalb normaler Grenzen zu halten."

Freiburg i. Br., Lehenstr. 36 II. 